\title{
The FKBP5 polymorphism rs1360780 influences the effect of an algorithm-based antidepressant treatment and is associated with remission in patients with major depression
}

\author{
Thomas J Stamm ${ }^{1 *}$, Carina Rampp ${ }^{2 *}$, Katja Wiethoff1, Julia Stingl ${ }^{3}$,

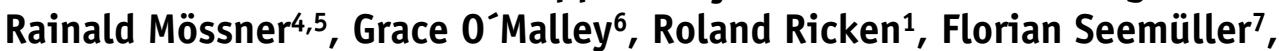 \\ Martin Keck², Robert Fisher ${ }^{8}$, Wolfgang Gaebel ${ }^{9}$, Wolfgang Maier ${ }^{4}$, \\ Hans-Jürgen Möller ${ }^{7}$, Michael Bauer ${ }^{10}$ and Mazda Adli ${ }^{1}$
}

\begin{abstract}
Objective: The FKBP5-gene influences the HPA-system by modulating the sensitivity of the glucocorticoid receptor (GR). The polymorphism rs1360780 has been associated with response in studies with heterogeneous antidepressant treatment. In contrast, several antidepressant studies with standardized antidepressant treatment could not detect this effect. We therefore compared patients with standardized vs naturalistic antidepressant treatment to (a) investigate a possible interaction between FKBP5-genotype and treatment mode and (b) replicate the effect of the FKBP5-genotype on antidepressant treatment outcome.

Methods: A total of 298 major depressive disorder (MDD) inpatients from the multicentred German project and the Zurich Algorithm Project were genotyped for their FKBP5 status. Patients were treated as usual $(n=127)$ or according to a standardized algorithm ( $n=171)$. Main outcome criteria was remission (Hamilton Depression Rating Scale-21<10).

Results: We detected an interaction of treatment as usual (TAU) treatment and C-allele with the worst outcome for patients combining those two factors $(H R=0.46 ; p=0.000)$. Even though $C$-allele patients did better when treated in the structured, stepwise treatment algorithm (SSTR) group, we still could confirm the influence of the FKBP5-genotype in the whole sample (HR=0.52; $p=0.01)$.

Conclusions: This is the first study to show an interaction between a genetic polymorphism and treatment mode. Patients with the C-allele of the rs1360780 polymorphism seem to benefit from a standardized antidepressant treatment.
\end{abstract}

Keywords

Depression, algorithm, pharmacogenetics

\section{Introduction}

Dysregulation of the stress hormone system in depression is one of the most robust findings in the landscape of biological psychiatry research (Holsboer, 2000). Hypothalamic-pituitary-adrenal (HPA) axis dysfunction and abnormalities in the serotonergic system could be connected to mood disorders and suicidal behaviour (Pompili et al., 2010). Regeneration of the HPAsystem is regarded as a prerequisite for obtaining a stable remission following treatment with antidepressants (Holsboer and Barden, 1996; Papiol et al., 2007). The FKBP5-gene influences

\footnotetext{
${ }^{1}$ Department of Psychiatry and Psychotherapy, Charité Universitätsmedizin Berlin, Berlin, Germany

${ }^{2}$ Max Planck Institute for Psychiatry, München, Germany

${ }^{3}$ Department of Pharmacology of Natural Products and Clinical

Pharmacology, University of Ulm, Ulm, Germany

${ }^{4}$ Department of Psychiatry and Psychotherapy, University Hospital Bonn, Bonn, Germany

${ }^{5}$ Department of Psychiatry and Psychotherapy, University of Tübingen, Tübingen, Germany

${ }^{6}$ School of Psychology, University College Dublin, Dublin, Ireland 'Department of Psychiatry and Psychotherapy, University Hospital Ludwig-Maximilian University, München, Germany
}

the function of the HPA-system by modulating the sensitivity of the glucocorticoid receptor (GR). Elevated levels of FKBP5 lead to a decreased GR sensitivity resulting in decreased feedback sensitivity of the system (Vermeer et al., 2003). Several functional polymorphisms have been described for the FKBP5 gene. TT-carriers of the polymorphism rs 1360780 in the FKBP5 gene presented FKBP5 levels which were twice as high as C-allele carriers in vitro (Binder et al., 2004). Previous studies have demonstrated a significantly higher likelihood of response to

\footnotetext{
${ }^{8}$ City and Hackney Centre for Mental Health, East London NHS Foundation Trust, London, UK

${ }^{9}$ Department of Psychiatry and Psychotherapy, Heinrich-HeineUniversität, Düsseldorf, Germany

${ }^{10}$ Department of Psychiatry and Psychotherapy, University Hospital Carl Gustav Carus, Dresden, Germany
}

*These authors contributed equally.

\section{Corresponding author:}

Thomas Stamm, Department of Psychiatry and Psychotherapy, Charite Universitaetsmedizin Berlin, Chariteplatz 1, 10117 Berlin, Germany. Email: thomas.stamm@charite.de 
antidepressant treatment for TT-homozygotes (Binder et al., 2004) respective T-allele carriers (Kirchheiner et al., 2008). Indeed, a recent meta-analysis confirmed a small advantage of T-allele carriers in a stratified analysis of patients of Central European origin (Niitsu et al., 2013). In contrast to these findings, however, a handful of studies have failed to support the hypothesis of the influence of rs1360780 on antidepressant response (Lekman et al., 2008; Papiol et al., 2007; Sarginson et al., 2010; Tsai et al., 2007). Those studies supporting the hypothesis and those rejecting it differed regarding the implementation of naturalistic vs standardized antidepressant treatment The supportive studies were naturalistic in their design; using a variety of antidepressant agents and combination therapies whereas the negative studies used a standardized selective serotonin reuptake inhibitor (SSRI) monotherapy. In summary, Table 1 gives an overview of all relevant studies on the association of the rs1360780 and treatment outcome. The German Algorithm Project Phase 3 (GAP3) as well as the Zurich algorithm-based step-by-step plan described in this report both evaluated a structured, stepwise treatment regime (SSTR) using different subsequent treatment strategies in inpatients with depression. The objective of these studies was to prove superiority of a highly-standardized algorithm-based treatment over treatment as usual (TAU) (Adli et al., unpublished).

Therefore, we used treatment data from these projects to investigate the following main questions:

1. Is there an interaction of the polymorphism in the FKBP5 gene with naturalistic vs standardized treatment?

2. Is there an effect of the polymorphism in the FKBP5 gene on antidepressant response in our total sample taking into account the whole treatment process?

\section{Patients and methods}

\section{Sample}

The total sample consisted of 298 inpatients of Central European origin with a major depressive disorder (MDD) who were recruited from the German Competence Network on Depression/ German Algorithm Project (GAP3) (Adli et al., 2003; Seemüller et al., 2010) and the Zurich Algorithm Project (Montani et al., 2007). Of 593 patients admitted with a depressive syndrome to one of the participating hospitals between 2000-2005, 475 were enrolled in the GAP3 and Zurich Algorithm Project of whom 429 were eligible for further analysis:. Pharmacogenetics analysis was conducted only if an additional informed consent (additional to the clinical study) was given. As a consequence, pharmacogenetics analysis was conducted with 298 out of 429 (69.6\%) patients. Within the German Competence Network on Depression, the GAP3 compared a SSTR against treatment as usual (TAU). The study was run across multiple psychiatric settings (six academic and three non-academic psychiatric hospitals) (Adli et al., 2003). The Zurich study was the Swiss equivalent to GAP3, and given its clear correspondence to GAP3 in its implementation and therapeutic goals (Montani et al., 2007) it was included in the research to increase the study's sample size. Both studies aimed at evaluating the benefits of algorithm-guided treatment of depression compared to TAU (Adli et al., 2006). To allow inclusion of representative clinical populations the following inclusion criteria were applied: a major depressive episode according to DSM-IV (American Psychiatric Association, 2000) criteria, and a Hamilton Depression Rating Scale (HDRS-21) score $\geqslant 15$. Age at inclusion was 18-70 years. Additionally, written informed consent had to be obtained, after the procedure had been fully explained. Exclusion criteria were: diagnosis of bipolar disorder; depression caused by other medical conditions; currently being pregnant or breastfeeding; any other prior antidepressant treatment that could not be discontinued; or acute and severe suicidality. Other axis I or II disorders were allowed if they were not the primary diagnosis. All intervention study arms in SSTR initially involved antidepressant monotherapy. All patients admitted to either of the participating centres were systematically assessed for eligibility and randomized to the SSTR or TAU group. The SSTR group ( $n=171)$ was treated according to a fixed algorithm starting with an antidepressant monotherapy. Within the three SSTR groups, all participants began with any one of four different antidepressants chosen to represent common pharmacological classes registered in Germany at the time of the protocol initiation. Allowed antidepressants were venlafaxine (SNRI, $225 \mathrm{mg} /$ day), sertraline (SSRI, $100 \mathrm{mg}$ / day), amitriptyline (TCA, $150 \mathrm{mg}$ /day) or reboxetine (noradrenaline reuptake inhibitor (NARI), $8 \mathrm{mg}$ /day), representing different antidepressant classes. Treating physicians could choose between those four medications, but had to maintain the dosage for at least four weeks, in cases of partial response for six weeks. In cases of non-response to monotherapy, patients continued with the secondstep strategy according to initial randomization: augmentation with lithium, dose escalation, or change of the antidepressant. In cases of continued failure to response to treatment, patients progressed to the next step in the algorithm after a given time period until electro convulsive therapy (ECT). The TAU group $(n=127)$ was treated naturalistically as described elsewhere (Adli et al., 2003), but underdosage of antidepressants was prohibited. The institutional local review boards approved the study design in all study centres. Trial Registration: http://www.germanctr.de/ Identifier: DRKS00000161.

\section{Procedure}

Treatment response was assessed using the HDRS-21 at baseline and again every two weeks during treatment. Patients were rated by independent study investigators who were uninvolved in the clinical management of patients and who were blinded to pharmacogenetic analysis. In the case of non-improvement after four weeks of treatment (indicated by HDRS-21 reduction $<30 \%$ ), patients progressed to the next step of the algorithm. In case of partial response (HRDS-21 reduction of $30-50 \%$ ), the current strategy was prolonged for further two weeks. Therefore, the maximum length of time for antidepressant monotherapy was six weeks. Remission was defined as achieving a score $<10$ on the HDRS-21. Across the team of study investigators, inter-rater reliability on the HDRS was assessed at frequent training sessions.

Study staff and patients were masked to the randomization code until inclusion assessment was finished. A systematic interview captured baseline clinical and socio-demographic features of the sample. Clinical diagnoses were confirmed with the Structured Clinical Interview for DSM-IV (SCID-I). Treatment outcome was assessed every two weeks ( \pm three days) by nonmasked research staff who were uninvolved in treatment. The primary outcome based on the HRDS-21 was time to remission 
Table 1. FKBP5 rs1360780 and antidepressant response: research overview.

\begin{tabular}{|c|c|c|c|c|}
\hline Authors & Sample & Ethnicity & Antidepressant treatment & Main results for FKBP5 rs 1360780 \\
\hline Binder et al., 2004 & $\begin{array}{l}294 \text { (MDD/DYS/BP) } \\
\text { inpatients }\end{array}$ & Central European origin & $\begin{array}{l}\text { Naturalistic: heterogeneous/ } \\
\text { combinations } 2 \text { and } 5 \text { weeks }\end{array}$ & $\begin{array}{l}\text { Better and faster response for } \\
\text { TT-genotype }\end{array}$ \\
\hline Papiol et al., 2007 & 159 MDD outpatients & Spanish & $\begin{array}{l}\text { Citalopram monotherapy for } \\
\text { up to } 12 \text { weeks }\end{array}$ & No association \\
\hline Tsai et al., 2007 & $125 \mathrm{MDD} / \mathrm{DYS}$ & Taiwanese & $\begin{array}{l}\text { Fluoxetine monotherapy } 4 \\
\text { weeks }\end{array}$ & No association \\
\hline Kirchheiner et al., 2008 & 179 MDD/BP inpatients & Central European origin & $\begin{array}{l}\text { Naturalistic: heterogeneous/ } \\
\text { combinations } 3 \text { weeks }\end{array}$ & $\begin{array}{l}\text { Better response in T-allele carriers; } \\
\text { worst response in CC-genotype } \\
\text { combination therapy patients }\end{array}$ \\
\hline Lekman et al., 2008 & $\begin{array}{l}1809 \text { (1256 subsample) } \\
\text { outpatients }\end{array}$ & $\begin{array}{l}\text { Mixed non-Hispanic } \\
\text { White }\end{array}$ & $\begin{array}{l}\text { Citalopram monotherapy } 14 \\
\text { weeks }\end{array}$ & $\begin{array}{l}\text { No association in whole sample; } \\
\text { trend for higher remission rates in } \\
\text { TT non-Hispanic White }\end{array}$ \\
\hline Sarginson et al., 2010 & $\begin{array}{l}246 \text { MDD (geriatric) } \\
\text { outpatients }\end{array}$ & $\begin{array}{l}20 \text { mixed } 226 \text { non- } \\
\text { Hispanic white }\end{array}$ & $\begin{array}{l}\text { Paroxetine or mirtazapine } \\
\text { monotherapy } 8 \text { weeks }\end{array}$ & No association \\
\hline Horstmann et al., 2010 & 374 MDD inpatients & Central European origin & $\begin{array}{l}\text { Naturalistic: heterogeneous/ } \\
\text { combination } 5 \text { weeks }\end{array}$ & $\begin{array}{l}\text { Gene } \times \text { Gene interaction with best } \\
\text { outcome for TT-genotype } \times \text { GRIK } 4 \\
\text { GG-genotype }\end{array}$ \\
\hline $\begin{array}{l}\text { Niitsu et al., 2013, } \\
\text { meta-analysis }\end{array}$ & $\begin{array}{l}2194 \text { ( } 1405 \text { subsample } \\
\text { with Central European } \\
\text { Origin) }\end{array}$ & Mixed & Meta-analysis & $\begin{array}{l}\text { No association in whole sample; } \\
\text { better response for T-allele } \\
\text { carriers in Central Europeans }\end{array}$ \\
\hline
\end{tabular}

MDD: major depressive disorder; BP: bipolar disorder; DYS: dysthymia.

(nine or less on the HAMD-21). After the completion of the inclusion assessment, patients, physicians and outcome assessors were not blinded to the treatment allocation.

For the present study, two different treatment periods were analysed according to the algorithm: The first period corresponded to the duration of antidepressant monotherapy in the algorithm arms, and could last up to six weeks. The second analysis reflects the whole treatment process from inclusion until end of study, i.e. up to a maximum of 14 weeks. The end-point of the study was remission or drop-out due to violation of the algorithm protocol, discharge from hospital or withdrawal of consent.

\section{Statistical analysis}

Statistical analysis was performed using SPSS 19.0. Descriptive statistics were performed to describe demographic and clinical characteristics of the study sample. Group comparisons were performed using the $\chi^{2}$ test for categorical variables, the univariate analysis of variance (ANOVA) for continuous variables and the independent $t$-test for the comparison of two groups. The HardyWeinberg equilibrium was tested with the $\chi^{2}$ test. The central statistical method used was the Cox regression survival analysis which facilitated the inclusion of censored cases. Calculations were completed using the forward LR method. In addition to our primary independent variable genotype, categorical covariates controlled for included study centre, treatment mode (standardized vs naturalistic), gender, psychotic symptoms and severity. Continuous covariates entered into the analyses included age and HDRS-21 baseline. For each observation period, all 298 patients were entered into the analysis, during which time weekly patient remissions or drop outs were registered in the dataset.

To examine the interaction between genotype and treatment mode, the product term genotype $\times$ treatment mode was included in the Cox regression analysis after including genotype and treatment mode individually: genotype+treatment mode + genotype $\times$ treatment mode.

Other exploratory analyses were performed to investigate the effects of different treatment strategies including class of antidepressants, number of treatment strategies and number of psychotropic medications. For both interaction analyses, the genotype variable was pooled in $\mathrm{T} / \mathrm{T}$ vs $\mathrm{C}$-allele carriers. For all analyses, a two-tailed $p$ value less than 0.05 was used as indicator for statistical significance.

\section{Genotyping}

For genotyping the rs1360780 variants in the FKBP5 gene the RealTime PCR method (TaqMan) was applied according to the protocol described by Kirchheiner et al. (2008).

\section{Results}

The total sample size comprised 298 patients, 187 women and 111 men, who had been genotyped for the FKBP5 rs1360780 variants. Genotype frequencies as well as distribution of demographic and clinical data are given in Table 2. No significant differences were detected between the genotypes. Likewise no significant differences were found between the two treatment modes (SSTR vs TAU) concerning demographic and baseline clinical characteristics. There was a trend for shorter treatment duration in TT genotype patients reaching significance when $\mathrm{C}$-allele carriers were pooled. Allele frequencies were in the Hardy-Weinberg equilibrium. Antidepressant substance classes were registered for 278 patients and did not differ significantly between genotypes. Comparing treatment factors between SSTR and TAU revealed significant differences for treatment duration, 
Table 2. Clinical and sociodemographic characteristics.

\begin{tabular}{|c|c|c|c|c|c|c|c|c|}
\hline & \multirow[t]{2}{*}{$n=298$} & \multicolumn{3}{|l|}{ SSTR $(n=171)$} & \multicolumn{3}{|l|}{ TAU $(n=127)$} & \multirow{2}{*}{$\begin{array}{l}\text { Statistics } \\
\text { SSTR vs TAU }\end{array}$} \\
\hline & & Tा $n=11$ & CT $n=68$ & CC $n=92$ & $\pi \mathrm{T} n=12$ & CT $n=52$ & CC $n=63$ & \\
\hline Gender & 298 & & & & & & & $\chi^{2}$ test n.s. \\
\hline Female & 187 & 6 & 40 & 64 & 9 & 30 & 38 & \\
\hline Male & 111 & 5 & 28 & 28 & 3 & 22 & 25 & \\
\hline $\begin{array}{l}\text { Severity of } \\
\text { depressive syndrome }\end{array}$ & 298 & & & & & & & $\chi^{2}$ test n.s. \\
\hline Light & 4 & 0 & 1 & 1 & 0 & 0 & 2 & \\
\hline Moderate & 85 & 3 & 25 & 25 & 4 & 15 & 13 & \\
\hline Severe & 185 & 7 & 38 & 61 & 8 & 30 & 41 & \\
\hline $\begin{array}{l}\text { With psychotic } \\
\text { symptoms }\end{array}$ & 24 & 1 & 4 & 5 & 0 & 7 & 7 & \\
\hline Age (years) & 298 & $39.91(9.07)$ & $45.79(12.32)$ & $45.26(12.67)$ & $43.08(10.88)$ & $41.81(12.07)$ & $44.56(11.33)$ & ANOVA n.s. \\
\hline $\begin{array}{l}\text { Number of prior } \\
\text { episodes }\end{array}$ & 234 & $0.86(0.9)$ & $1.52(1.94)$ & $1.34(2.53)$ & $2.91(4.74)$ & $1.7(2.57)$ & $1.85(2.46)$ & ANOVA n.s. \\
\hline HRDS baseline & 298 & $24.27(4.56)$ & $25.12(5.06)$ & $25.14(5.59)$ & $23.83(6.48)$ & $23.87(4.78)$ & $26.75(6.68)$ & ANOVA n.s. \\
\hline $\begin{array}{l}\text { Duration in study } \\
\text { (weeks) }\end{array}$ & 298 & $4.55(1.75)$ & $5.66(2.84)$ & $5.26(3.23)$ & $4.17(3.13)$ & $6.54(3.88)$ & $6.25(4.07)$ & $\begin{array}{l}\text { ANOVA } p<0.05 \\
(T T \text { vs } C C+C T) p<0.05\end{array}$ \\
\hline Class of ADs & 278 & & & & & & & $\chi^{2}$ test $p<0.001$ \\
\hline SSNRI & 103 & 6 & 32 & 44 & 1 & 9 & 11 & \\
\hline SSRI & 55 & 2 & 18 & 18 & 1 & 9 & 7 & \\
\hline Tricyclic AD & 35 & 1 & 9 & 12 & 2 & 2 & 9 & \\
\hline NaSSA & 24 & 1 & 1 & 6 & 2 & 8 & 6 & \\
\hline NARI & 9 & 0 & 2 & 5 & 0 & 1 & 1 & \\
\hline MAO inhibitors & 3 & 0 & 0 & 0 & 0 & 1 & 2 & \\
\hline AD combinations & 54 & 0 & 2 & 6 & 5 & 17 & 24 & \\
\hline $\begin{array}{l}\text { Number of } \\
\text { psychotropic } \\
\text { medications }\end{array}$ & 278 & $1.30(0.48)$ & $1.44(0.85)$ & $1.80(1.24)$ & $1.73(0.9)$ & $2.89(1.81)$ & $2.45(1.41)$ & ANOVA $p<0.001$ \\
\hline $\begin{array}{l}\text { Number of } \\
\text { treatment strategies }\end{array}$ & 278 & $1.20(0.42)$ & $1.42(0.87)$ & $1.71(1.13)$ & $1.55(0.82)$ & $2.49(1.31)$ & $2.02(1.13)$ & ANOVA $p<0.001$ \\
\hline
\end{tabular}

AD: antidepressant; ANOVA: analysis of variance; HRDS: Hamilton-Rating-Depression-Scale; MA0-inhibitors: monoaminoxidase inhibitors (tranylcypromine, moclobemide); NARI: noradrenaline reuptake inhibitor; NaSSA: noradrenergic and specific serotonergic antidepressant (mirtazapine, mianserine); SSNRI: selective serotonin/noradrenaline reuptake inhibitor; SSRI: selective serotonin reuptake inhibitor; TAU: treatment as usual.

the number of prescribed psychotropic medications, number of applied treatment strategies and the distribution of the prescribed antidepressant substance classes in both treatment modes (see Table 2). Mean duration of the current depressive episode was 22 weeks (standard deviation (SD) 39.8) for the whole sample with a duration of 20.9 weeks (SD 43.9, $n=146$ ) in the SSTR group and 23.8 weeks (SD 32.7, $n=90$ ) in the TAU group (ANOVA; n.s.). Patients in the SSTR group had a shorter stay in the study, had fewer psychotropic medications and fewer changes of treatment strategy. Antidepressant monotherapy with SSRI or selective serotonin/noradrenaline reuptake inhibitor (SSNRI) were found much more often in SSTR group while TAU patients were treated more frequently with antidepressant combinations. These findings contrast to the fact that treatment groups did not differ regarding depression severity at baseline measured by HRDS-21 score or number of prior depressive episodes (see Table 2). Overall remission rate was $39.9 \%$ after the first six weeks of treatment and increased to $54.7 \%$ at the end of the study. The steps to monotherapy, lithium augmentation and monoaminoxidase (MAO) inhibitors resulted in high remission rates or significant clinical improvement leading to hospital discharge. Consequently, ECT was not applied during the trial. Remission rates were significantly different between both treatment modes with $66.1 \%$ for SSTR and only $39.3 \%$ for TAU $\left(\chi^{2}\right.$; $p<0.001)$. Remission rates for the FKBP5 genotypes differed significantly after the first treatment step (TT $60.9 \%$ vs $\mathrm{CT} / \mathrm{C} / \mathrm{C}$ $38.2 \% ; \chi^{2} ; p=0.03$ ) but not after the entire treatment process (TT $69.6 \%$ vs $\mathrm{CT} / \mathrm{CC} 53.5 \% ; \chi^{2} ; p$ n.s.). Interestingly, remission rates for $\mathrm{CC} / \mathrm{CT}$ allele carriers were significantly higher in SSTR compared to CC/CT patients in TAU (64.4\% SSTR vs $38.8 \%$ TAU; $\left.\chi^{2} ; p<0.001\right)$, but not in TT-genotype patients, supporting the hypothesized interaction between FKBP5 genotype and treatment mode.

\section{Interaction between genotype and treatment mode}

The main study objective was to examine the potential interaction between genotype and treatment mode with regard to the treatment outcome across the entire study period. Treatment mode had a significant influence on treatment outcome in C-allele 
(a)

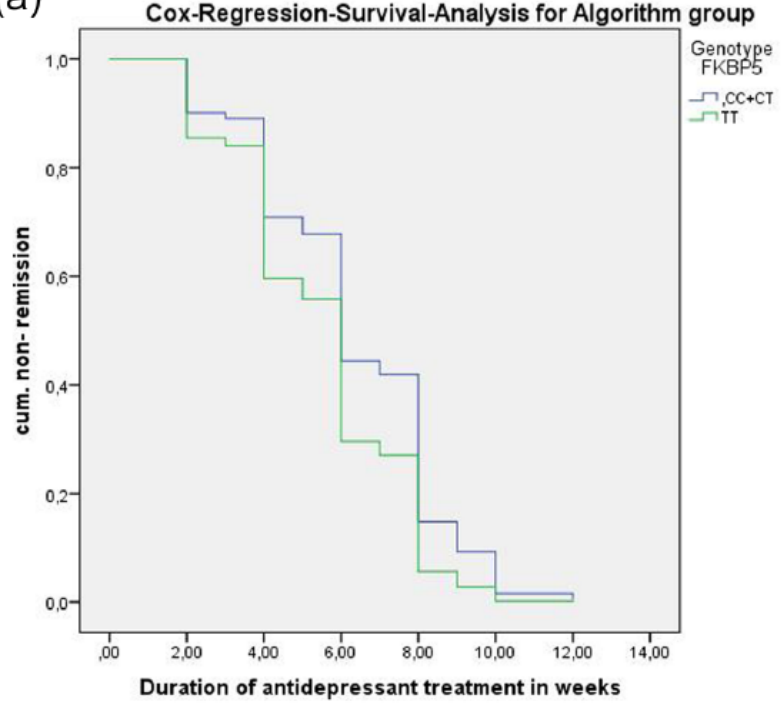

(b)

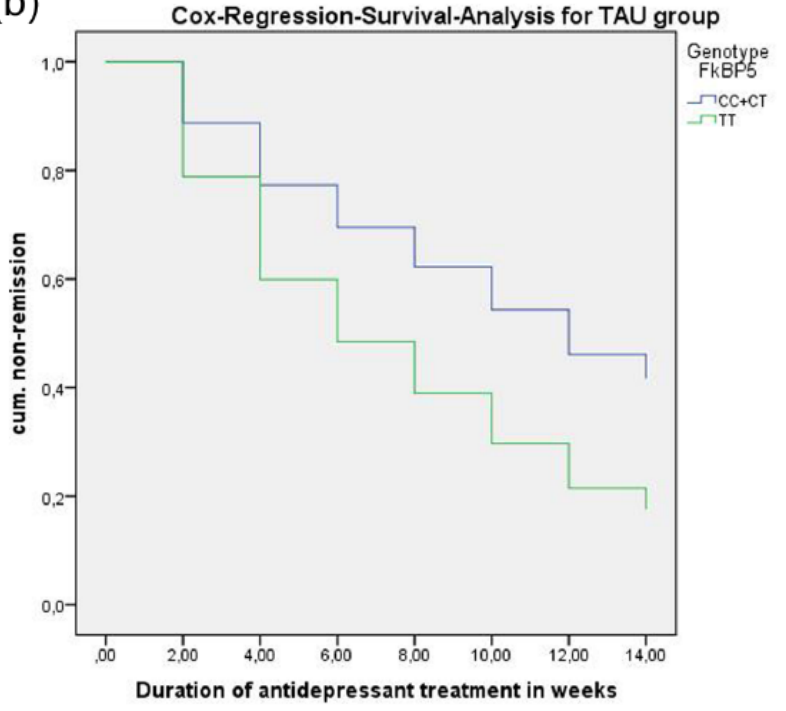

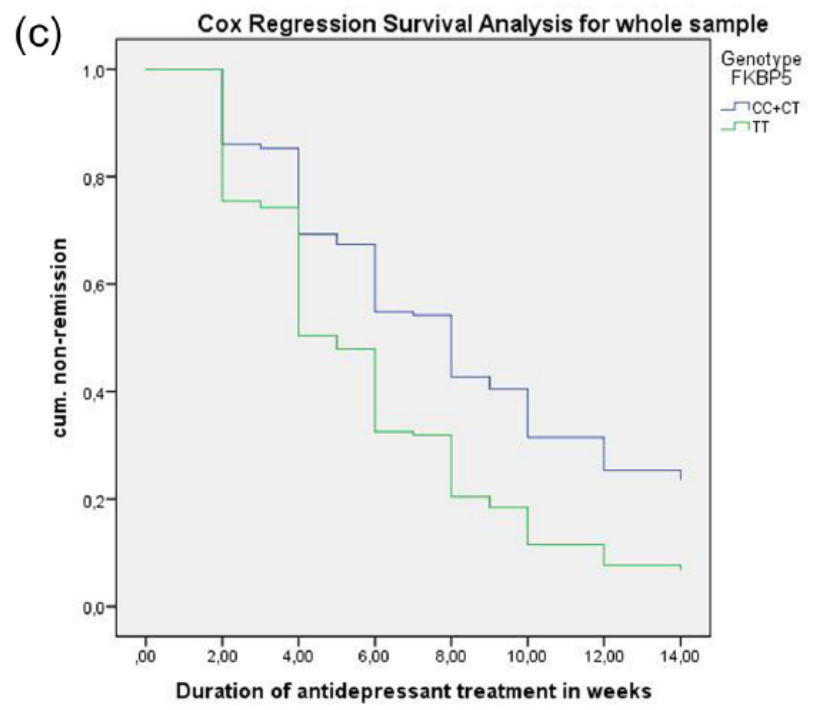

Figure 1. Cox regression survival analysis for FKBP5 genotype (all other factors kept constant): in (a) SSTR; (b) treatment as usual (TAU); and (c) in the whole sample.

carriers but not in TT-homozygous patients. We observed a significant interaction in terms of a synergistic effect between naturalistic treatment (TAU) and the C-allele of the FKBP5 gene. TAU patients carrying the $\mathrm{C}$-allele showed the least effective treatment outcome. The product variable indicating the interaction effect was significant for remission $(p<0.001$; Hazard Ratio $(\mathrm{HR})=0.46$ ); genotype and treatment mode both lost their respective significances in the interaction analysis. These findings suggest a mutual potentiating effect of naturalistic treatment and the C-allele. No synergistic effect of standardized treatment and TT-genotype was found.

To illustrate the interaction effect, we conducted separate survival analyses for both treatment modes (see Figure 1 (a)-(c)): In the SSTR group, the FKBP5 genotype did not contribute significantly to treatment outcome whereas in the TAU group a large difference between $\mathrm{CC} / \mathrm{CT}$ and TT allele carriers could be shown (HR $0.46 ; p<0.05$ ).

We additionally conducted an exploratory analysis on which of the treatment factors might have led to the unfavourable outcome for TAU/C-allele patients. This entailed the inclusion of the significantly different treatment factors (i.e. number of prescribed psychotropic medications, number of treatment strategies and antidepressant substance classes) into our cox regression model together with the $\mathrm{C}$ - allele genotype. Two out of these three factors emerged as significant contributors: Patients with less than 3 prescribed psychotropic medications (HR $4.5 ; p<0.001)$ and 2 treatment changes at the most (HR 4.1; $<<0.001)$ yielded the best treatment outcome. Lastly, no interaction between genotype and substance classes with regard to the treatment outcome was found. This suggests that the influence of the genotype on treatment 
outcome does not differ significantly across different antidepressants. Study centre did not influence outcome in our sample.

\section{Influence of genotype on remission in the whole sample}

Regarding the second main objective of the study we found a significant influence of genotype on remission during the first six weeks (antidepressant monotherapy in SSTR) and over the whole observation period. After up to six weeks of antidepressant treatment we already detected a significant better outcome for TT-allele patients $(\mathrm{HR}=1.89 ; p<0.03)$. For the whole treatment process of up to 12 weeks, we confirmed the findings of other previous studies involving patients of Central European origin. We observed a higher probability of achieving remission in TT-genotype, while CC- and CT-genotype patients were less likely to achieve remission $(\mathrm{HR}=0.52 ; p=0.01)$. Besides the FKBP5 genotype, only baseline depression scores $(\mathrm{HR}=0.05$; $p<0.01)$ and treatment mode $(\mathrm{HR}=2.0 ; p<0.01)$ prevailed as significant contributors for achieving remission.

\section{Discussion}

To the best of our knowledge, the present study reflects the first research to demonstrate a significant interaction between the FKBP5 genotype at rs 1360780 and treatment mode in a sample of depressed inpatients of Central European origin. It is also the first study to examine the association of the polymorphism with remission during an entire multi-step treatment process over a longer period of time comprising different antidepressant strategies. We successfully showed that the influence of genotype persists over a long treatment process when applying different antidepressant strategies. C-allele carriers had a significantly worse outcome when treated naturalistically and showed improved remission rates when treated according to a systematic treatment algorithm. In contrast, TT-allele carriers showed a superior treatment response across both SSTR and TAU treatment conditions. Combining our algorithm and the TAU group, we were able to confirm the results of pre-existing studies and a recent meta-analysis of a more favourable treatment outcome in T-allele patients (Binder et al., 2004; Horstman et al., 2010; Kirchheiner et al., 2008; Niitsu et al., 2013). Contradictory findings exist (Lekman et al., 2008; Sarginson et al., 2010; Tsai et al., 2007) and in addition to the risk of non-replication of single nucleotide polymorphism association studies, a number of reasons may be responsible for inconsistencies in the body of research to date: Firstly, patient ethnicity varied with Asian (Tsai et al., 2007), Spanish (Papiol et al., 2007), mixed Afro- and non-Hispanic white-American (Lekman et al., 2008; Sarginson et al., 2010) and Central European patients (Binder et al., 2004; Horstmann et al., 2010; Kirchheiner et al., 2010) being analysed for their FKBP5 status. Therefore, the meta-analysis by Niitsu et al. (2013) stratified for ethnicity and could only confirm a small but significant superior response for T-allele carriers in patients of Central European origin. Secondly, studies were different regarding important clinical variables such as depression severity, diagnosis, duration of treatment and choice of the antidepressant strategy. While the largest study by Lekman et al. (2008), as part of the Star*d project, involved outpatients exclusively, Binder et al. (2004), Kirchheiner et al. (2010) and
Horstmann et al. (2010) and the present study included inpatients only. In some studies, patients with bipolar depression or dysthymia were included; others only integrated patients with unipolar depression and a severity cut-off. Furthermore, our data point to another important difference potentially relevant for inconsistencies of the published studies on the FKBP5 influence on antidepressant response: All of the studies lacking evidence for the influence of genotype used a homogenous treatment design, mostly with SSRI monotherapy as the single exclusive treatment (Lekman et al., 2008; Papiol et al., 2007; Tsai et al., 2007). Only in the hypothesis-rejecting study by Sarginson et al. (2010), were both mirtazapine and the SSRI paroxetine respectively studied as monotherapeutic agents. In the hypothesis-supporting studies, treatment was allowed to be naturalistic with various antidepressants and treatment combinations. Given that our data facilitated the direct comparison of the effects of naturalistic vs structured antidepressant treatment; we may have identified a possible explanation for this effect.

We found a synergistic effect between TAU and CT- resp. CC-genotype implicating that carriers of the 'unfavourable' C-allele may particularly benefit from SSTR. This standardized treatment may compensate for their disadvantage in particular by potentially engaging those patients in prolonged antidepressant monotherapy. For the T/T-genotype, the treatment mode seems to be less crucial.

In essence, SSTR differed from TAU by exemplifying clear, sequential therapy guidelines and by diligently evaluating treatment response at critical decision points as the basis for a standardized treatment decision process (Adli et al., 2003; Bauer et al., 2009). By its structured treatment protocol, SSTR prevents premature treatment changes, polypharmacy and unstructured application of treatment strategies. Patients with the unfavourable FKBP5 genotype seem to benefit particularly from this treatment optimization.

The underlying biological mechanisms of what precisely characterizes the difference in treatment response between the FKBP5 genotypes are still uncertain. FKBP-5 acts as a co-chaperone that modulates sensitivity of the GR (Zannas et al., 2015). In particular, in vitro experiments have shown that FKBP5 reduces interaction of the GR complex with the transport protein dynein, delays nuclear translocation of the GR, and decreases GR-dependent transcriptional activity (Wochnik et al., 2005) By modulating the HPA axis through GR signalling, FKBP5 seems to play an important relay function that is highly relevant for many patients with affective disorders. A number of studies could demonstrate a clear association of FKBP5 status with clinical markers of depression: After eight weeks of escitalopram or nortriptyline monotherapy, levels of mRNA of FKBP5 were significantly reduced in responders but not in non-responders (Cattaneo et al., 2013). Binder described a stronger dysregulation of the HPA-system in T-allele - compared to C-allele carriers - during a depressive episode (Binder, 2009). Due to a tighter interplay of FKBP5-expression and cortisol-levels in TT-carriers, their HPA-regulation seems to be less stable and more sensitive to environmental influences (Binder et al., 2004; Klengel et al., 2013). This might explain the superior response in TT-patients because antidepressants may more easily interfere with HPAsystem regulation as it has been shown that antidepressants reduce Corticotropin-releasing hormone and cortisol levels 
(Ising et al., 2007). The interplay of cortisol levels and FKBP5 expression in TT-patients might lead to a more rapid downregulation of FKBP5 due to a reduction of cortisol levels during antidepressant treatment and thus to higher sensitivity of GRs. In both healthy and depressed C-allele carriers the cortisol-induced FKBP5-expression seems to be less pronounced. Their HPA-system seems to be less reactive to environmental influences. The proportionally higher HPA-dysregulation during depression and the lower correlation of cortisol and FKBP5 levels in $\mathrm{C}$-allele carriers might contribute to explain their inclination to benefit from a standardized treatment. These factors lead to a slower and reduced interference of the HPAsystem with antidepressant drugs. This would increase the importance of exhausting the chosen treatment strategy in $\mathrm{C}$-allele carriers. Therefore, these patients seem particularly responsive to the application of a structured procedure. The lower probability of remission during the whole study period in $\mathrm{C}$-allele carriers suggests a higher tendency to treatment resistance in those patients, possibly also due to the described biochemical mechanisms.

The results of our study emphasize the importance of a structured and algorithm-guided antidepressant treatment, especially as patients disadvantaged because of their genotype benefit from a structured treatment. A standardized procedure may allow higher remission rates achieved in a shorter time with less medication and changes of treatment strategies. Additionally, a genetic disadvantage can be compensated by algorithm-guided treatment. The findings suggest that a standardized, quality-controlled treatment may to an extent compensate for a 'genetic disadvantage' in C-allele carriers. Hence, the treatment results for $\mathrm{C}$-allele carriers approach to those of TT-carriers in algorithm-guided treatment. This might explain why the influence of this genotype on treatment response could not be replicated in all studies. As mentioned above, the negative studies with regard to FKBP5 genotype had a strictly homogenous treatment design with a predefined (mostly SSRIbased) monotherapy (similar to our own SSTR group), whereas the studies in support of the influence of genotype had a heterogeneous treatment design (as was the case in our TAU group).

\section{Limitations}

The first limitation of this study is the relatively small sample size for a pharmacogenetic study. Secondly, although the decision to recruit patients for this study from two different projects for this study was justifiable given their identical operations and characteristics, it is a source of heterogeneity. On the one hand, the inclusion of patients treated with different substance classes, partly naturalistically and partly standardized, led to heterogeneity in participants' medical treatment. On the other hand, the consideration of all patients made it possible to obtain a sufficiently large sample size and thereby allowed further analysis concerning the treatment mode. In relation to the main objective of the study, the explanatory power of an interaction between genotype and treatment mode is restricted due to the low percentage of T/T-carriers: Only $7.72 \%$ of the whole sample were $\mathrm{T} / \mathrm{T}$-carriers. Besides the FKBP5 gene, other sources of genetic heterogeneity could be examined regarding their influence on treatment in antidepressant therapy. Possible candidate genes could be the SLC6A4, HTR2A, BDNF, GNB3, ABCD1 and cytochrome p450 genes (Fabbri and Serretti, 2015).
Our intentions with these research questions were purely exploratory in nature and as such may be considered preliminary and requiring further analysis in future. Future studies are particularly important to rule out the possibility that the independence of the treatment mode in TT-patients is not in fact due to a randomly distribution; this was not possible in our sample.

\section{Conclusion}

The influence of the FKBP5 polymorphism rs 1360780 was successfully replicated in the present study suggesting a predictive value of this single nucleotide polymorphism for antidepressant treatment outcome in moderate to severely depressed inpatients of Central European origin. The polymorphism might contribute to more individually-tailored therapeutic choices in the future. As the effect of genotype does not seem to differ considerably across substance classes, it is currently not possible to draw clear conclusions concerning the choice of an antidepressant. C-allele carriers particularly seem to benefit from a structured algorithmguided treatment which compensates their 'genetic disadvantage'. Better treatment outcomes in TT-patients seem to be mostly independent of the applied treatment mode.

\section{Acknowledgements}

The study was conducted within the German Research Network on Depression in 10 psychiatric departments throughout Germany (six academic and four non-academic centres): Charité Campus Mitte (CCM) Berlin (leading study site), Charité Campus Benjamin (CBF) Franklin Berlin, Ludwig Maximilians University Munich, University of HalleWittenberg, University of Dusseldorf, University of Heidelberg, St Hedwig Hospital Berlin, St Joseph Hospital Berlin, Vivantes AugusteViktoria (AVK) Hospital Berlin and District Hospital Gabersee. The authors wish to thank the clinical staff of all contributing study sites for their involvement.

\section{Declaration of Conflicting Interests}

The authors declared the following potential conflicts of interest with respect to the research, authorship, and/or publication of this article: Thomas Stamm has received speaker honoraria from Lundbeck and Bristol-Myers Squibb. He is a consultant to Servier.

Martin Keck has received speaker bureau honoraria from and pursued other continuing medical education activity for AstraZeneca Switzerland, Eli Lilly Switzerland, Lundbeck Switzerland and Zeller Medical Switzerland. He has received advisory panel payment from Lundbeck Switzerland.

Wolfgang Gaebel is Member of the Faculty of the Lundbeck International Neuroscience Foundation. He has received symposia support from Janssen-Cilag GmbH, Neuss, Lilly Deutschland GmbH, Bad Homburg and Servier, Munich.

Hans-Jürgen Möller has received honoraria for lectures or for advisory activities or received grants from the following pharmaceutical companies: AstraZeneca, Eli Lilly, Janssen, Lundbeck, Pfizer, Schwabe, Servier, Otsuka and Takeda. He was president, or in the executive board, of the following organisations: The International College of Neuropsychopharmacology, European College of Neuropsychop-harmacology, World Federation of Societies of Biological Psychiatry, European Association of Psychiatry, and was chairman of the World Psychiatric Association-section on Pharmacopsychiatry.

Michael Bauer has received grant/research support from The Staney Medical Research Institute, National Alliance for Research on Schizophrenia and Depression, Deutsche Forschungsge-meinschaft, European Commission (FP7), American Foundation for Suicide Prevention, Bundesministerium für Bildung und Forschung (BMBF). 
$\mathrm{He}$ is/has been a consultant for Alkermes, AstraZeneca, Bristol-Myers Squibb, Ferrer Internacional, Janssen, Lilly, Lundbeck, Otsuka, Servier, Takeda and has received speaker honoraria from AstraZeneca, Bristol-Myers Squibb, GlaxoSmithKline. Lilly, Lundbeck, Otsuka and Pfizer.

Mazda Adli has received grant or research support from Aristo, Servier and Bristol-Myers Squibb; speaker honoraria from Deutsche Bank, the Johanniter Orden, East German Savings banks Association, Pusch Wahl Legal Lawyers, HRM Forum, Helios Media, Lundbeck, Bristol-Myers Squibb, Boehringer Ingelheim, Servier, Aristo, Viiv and Gilead. He has received travel grants from the Alfred Herrhausen Society, Lundbeck and Servier. He has been a consultant to Deutsche Bank, Bristol-Myers Squibb, Aristo, Merz and Lundbeck.

Carina Rampp, Katja Wiethoff, Julia Stingl, Rainald Mössner, Grace O'Malley, Roland Ricken, Florian Seemüller, Robert Fisher and Wolfgang Maier declared no potential conflicts of interest with respect to the research, authorship, and/or publication of this article.

\section{Funding}

The authors disclosed receipt of the following financial support for the research, authorship, and/or publication of this article: The German Algorithm Project Phase 3 (GAP3), was funded by the German Federal Ministry for Education and Research within the promotional emphasis 'German Research Network on Depression' (grant number: 01GI0218). Additional unrestricted research grants were received from Eli Lilly and Company, Janssen-Cilag, Pfizer Inc., Pharmacia, and WyethAyerst Laboratories, Inc.

\section{References}

Adli M, Bauer M and Rush AJ (2006) Algorithms and collaborative-care systems for depression: Are they effective and why? A systematic review. Biol Psychiatry 59: 1029-1038.

Adli M, Rush AJ, Möller HJ, et al. (2003) Algorithms for optimizing the treatment of depression: Making the right decision at the right time. Pharmacopsychiatry 36: S222-S229.

Adli M, Wiethoff K, Baghai TC, et al. How effective is algorithm-guided treatment for depressed inpatients? Results from the randomized multicenter German Algorithm Project 3 trial. Unpublished.

American Psychiatric Association (2000) Diagnostic and Statistical Manual of Mental disorders - (4th Ed. Text Revision). Washington, DC: American Psychiatric Association.

Bauer M, Pfennig A, Linden M, et al. (2009) Efficacy of an algorithmguided treatment compared with treatment as usual: A randomized, controlled study of inpatients with depression. J Clin Psychopharmacol 29: 327-333.

Binder EB (2009) The role of FKBP5, a co-chaperone of the glucocorticoid receptor in the pathogenesis and therapy of affective and anxiety disorders. Psychoneuroendocrinology 34: S186-S195.

Binder EB, Salyakina D, Lichtner P, et al. (2004) Polymorphisms in FKBP5 are associated with increased recurrence of depressive episodes and rapid response to antidepressant treatment. Nat Genet 36: 1319-1325.

Cattaneo A, Gennarelli M, Uher R, et al. (2013) Candidate genes expression profile associated with antidepressants response in the GENDEP study: Differentiating between baseline 'predictors' and longitudinal 'targets'. Neuropsychopharmacology 38: 377-385.

Fabbri C and Serretti A (2015) Pharmacogenetics of major depressive disorder: Top genes and pathways toward clinical applications. Curr Psychiatry Rep 17: 50.
Holsboer F (2000) The corticosteroid receptor hypothesis of depression. Neuropsychopharmacology 23: 477-501.

Holsboer F and Barden N (1996) Antidepressants and hypothalamicpituitary-adrenocortical regulation. Endocr Rev 17: 187-205.

Horstmann S, Lucae S, Menke A, et al. (2010) Polymorphisms in GRIK4, HTR2A, and FKBP5 show interactive effects in predicting remission to antidepressant treatment. Neuropsychopharmacology 35: $727-740$.

Ising M, Horstmann S, Kloiber S, et al. (2007) Combined dexamethasone/corticotropin releasing hormone test predicts treatment response in major depression - a potential biomarker? Biol Psychiatry 62: 47-54.

Kirchheiner J, Lorch R, Lebedeva E, et al. (2008) Genetic variants in FKBP5 affecting response to antidepressant drug treatment. Pharmacogenomics 9: 841-846.

Klengel T, Mehta D, Anacker C, et al. (2013) Allele-specific FKBP5 DNA demethylation mediates gene-childhood trauma interactions. Nat Neurosci 16: 33-41.

Lekman M, Laje G, Charney D, et al. (2008) The FKBP5-gene in depression and treatment response - an association study in the Sequenced Treatment Alternatives to Relieve Depression (STAR*D) Cohort. Biol Psychiatry 63: 1103-1110.

Montani T, Zumstein A, Lengen C, et al. (2007) Vergleich eines systematischen Therapiealgorithmus mit der Standardbehandlung nach freiem klinischem Ermessen bei Patienten mit einer depressiven Episode: Befunde aus der Zürcher Stufenplanstudie. Nervenarzt 78: 112.

Niitsu T, Fabbri C, Bentini F, et al. (2013) Pharmacogenetics in major depression: A comprehensive meta-analysis. Prog Neuropsychopharmacol Biol Psychiatry 45: 183-194.

Papiol S, Arias B, Gastó C, et al. (2007) Genetic variability at HPA axis in major depression and clinical response to antidepressant treatment. J Affect Disord 104: 83-90.

Pompili M, Serafini G, Innamorati M, et al. (2010) The hypothalamicpituitary-adrenal axis and serotonin abnormalities: A selective overview for the implications of suicide prevention. Eur Arch Psychiatry Clin Neurosci 260: 583-600.

Sarginson JE, Lazzeroni LC, Ryan HS, et al. (2010) FKBP5 polymorphisms and antidepressant response in geriatric depression. $\mathrm{Am}$ $J$ Med Genet B Neuropsychiatr Genet 153: 554-560.

Seemüller F, Riedel M, Obermeier M, et al. (2010) Outcomes of 1014 naturalistically treated inpatients with major depressive episode. Eur Neuropsychopharmacol 20: 346-355.

Tsai SJ, Hong CJ, Chen TJ, et al. (2007) Lack of supporting evidence for a genetic association of the FKBP5 polymorphism and response to antidepressant treatment. Am J Med Genet B Neuropsychiatr Genet 144: 1097-1098.

Vermeer H, Hendriks-Stegeman BI, van der Burg B, et al. (2003) Glucocorticoid-induced increase in lymphocytic FKBP51 messenger ribonucleic acid expression: A potential marker for glucocorticoid sensitivity, potency, and bioavailability. J Clin Endocrinol Metab 88: $277-284$.

Wochnik GM, Rüegg J, Abel GA, et al. (2005) FK506-binding proteins 51 and 52 differentially regulate dynein interaction and nuclear translocation of the glucocorticoid receptor in mammalian cells. J Biol Chem 280: 4609-4616.

Zannas AS, Wiechmann T, Gassen NC, et al. (2015) Gene-stress-epigenetic regulation of FKBP5: Clinical and translational implications. Neuropsychopharmacology Epub ahead of print 13 August 2015. DOI: 10.1038/npp.2015.235. 